\title{
Identification of the Aldo-Keto Reductase Responsible for D-Galacturonic Acid Conversion to L-Galactonate in Saccharomyces cerevisiae
}

\author{
Dorthe Rippert, Federica Linguardo (D), Andreea Perpelea, Mathias Klein and Elke Nevoigt * \\ Department of Life Sciences and Chemistry, Jacobs University Bremen gGmbH, 28759 Bremen, Germany; \\ d.ripper@@jacobs-university.de (D.R.); federica.linguardo@gmail.com (F.L.); \\ a.perpelea@jacobs-university.de (A.P.); m.klein@jacobs-university.de (M.K.) \\ * Correspondence: e.nevoigt@jacobs-university.de; Tel.: +49-421-200-3541; Fax: +49-421-200-3249
}

Citation: Rippert, D.; Linguardo, F.; Perpelea, A.; Klein, M.; Nevoigt, E. Identification of the Aldo-Keto Reductase Responsible for D-Galacturonic Acid Conversion to L-Galactonate in Saccharomyces cerevisiae. J. Fungi 2021, 7, 914. https://doi.org/10.3390/jof7110914

Academic Editor: Naseem A. Gaur

Received: 10 August 2021

Accepted: 25 October 2021

Published: 27 October 2021

Publisher's Note: MDPI stays neutral with regard to jurisdictional claims in published maps and institutional affiliations.

Copyright: () 2021 by the authors. Licensee MDPI, Basel, Switzerland. This article is an open access article distributed under the terms and conditions of the Creative Commons Attribution (CC BY) license (https:// creativecommons.org/licenses/by/ $4.0 /)$.

\begin{abstract}
D-galacturonic acid (D-GalUA) is the main constituent of pectin, a complex polysaccharide abundant in several agro-industrial by-products such as sugar beet pulp or citrus peel. During several attempts to valorise D-GalUA by engineering the popular cell factory Saccharomyces cerevisiae, it became obvious that D-GalUA is, to a certain degree, converted to L-galactonate (L-GalA) by an endogenous enzymatic activity. The goal of the current work was to clarify the identity of the responsible enzyme(s). A protein homology search identified three NADPH-dependent unspecific aldo-keto reductases in baker's yeast (encoded by GCY1, YPR1 and GRE3) that show sequence similarities to known D-GalUA reductases from filamentous fungi. Characterization of the respective deletion mutants and an in vitro enzyme assay with a Gcy1 overproducing strain verified that Gcy1 is mainly responsible for the detectable reduction of D-GalUA to L-GalA.
\end{abstract}

Keywords: D-galacturonic acid; L-galactonate; Saccharomyces cerevisiae; aldo-keto reductase; Gcy1

\section{Introduction}

The sugar acid D-galacturonic acid (D-GalUA) is, apart from D-glucose and L-arabinose, an abundant monomer in pectins. Pectins are complex polysaccharides found in various types of plant cell walls [1]. Several agro-industrial waste streams such as sugar beet pulp or citrus peels are rich in pectins [2]. These carbohydrate-abundant residues have been considered attractive feedstocks for industrial biotechnology, particularly because they are pre-treated during food processing and virtually do not contain any lignin, making their hydrolysis relatively efficient $[3,4]$. The valorisation of pectin-rich biomass residues has to include concepts for the conversion of D-GalUA into valuable products since the sugar acid makes up approximately $70 \%$ of pectins $[1,5,6]$.

Certain bacteria and fungi are able to naturally utilize D-GalUA [7]. There are two known bacterial pathways for D-GalUA catabolism [7]. The pathway for D-GalUA utilization in fungal organisms seems to be conserved among asco- and basidiomycetes; it consists of four enzymes [8] and was first described in the mould Trichoderma reesei [9] (Figure 1). The products of these catabolic reactions are pyruvate and glycerol, which are subsequently further broken down in the cell's central carbon metabolism. The first enzymatic step of the fungal D-GalUA pathway is the conversion of D-GalUA to L-galactonate (L-GalA) by a D-galacturonic acid reductase. L-GalA is further converted to 2-keto-3-deoxy-L-galactonate catalysed by an L-galactonate dehydratase. An aldolase splits the last intermediate into pyruvate and L-glyceraldehyde from which the latter compound is further converted to glycerol by an L-glyceraldehyde reductase [10,11].

With regard to the valorisation of pectin-rich biomass hydrolysates, fungal cell factories are generally preferred over bacteria due to a higher robustness towards the inhibitors present in such crude substrates. In fact, hydrolysates of pectin-rich biomass contain acetic 
acid and methanol from the acetylated and methyl-esterified polymers [5]. Both compounds are known as potent inhibitors of microbial growth and fermentation [5,12]. In hydrolysates of food waste streams, particularly acetic acid seems to reach concentrations which are inhibitory [13]. Last but not least, the abundant monomer D-GalUA is also a weak acid contributing to the potential stress in cells grown in such hydrolysates [14].

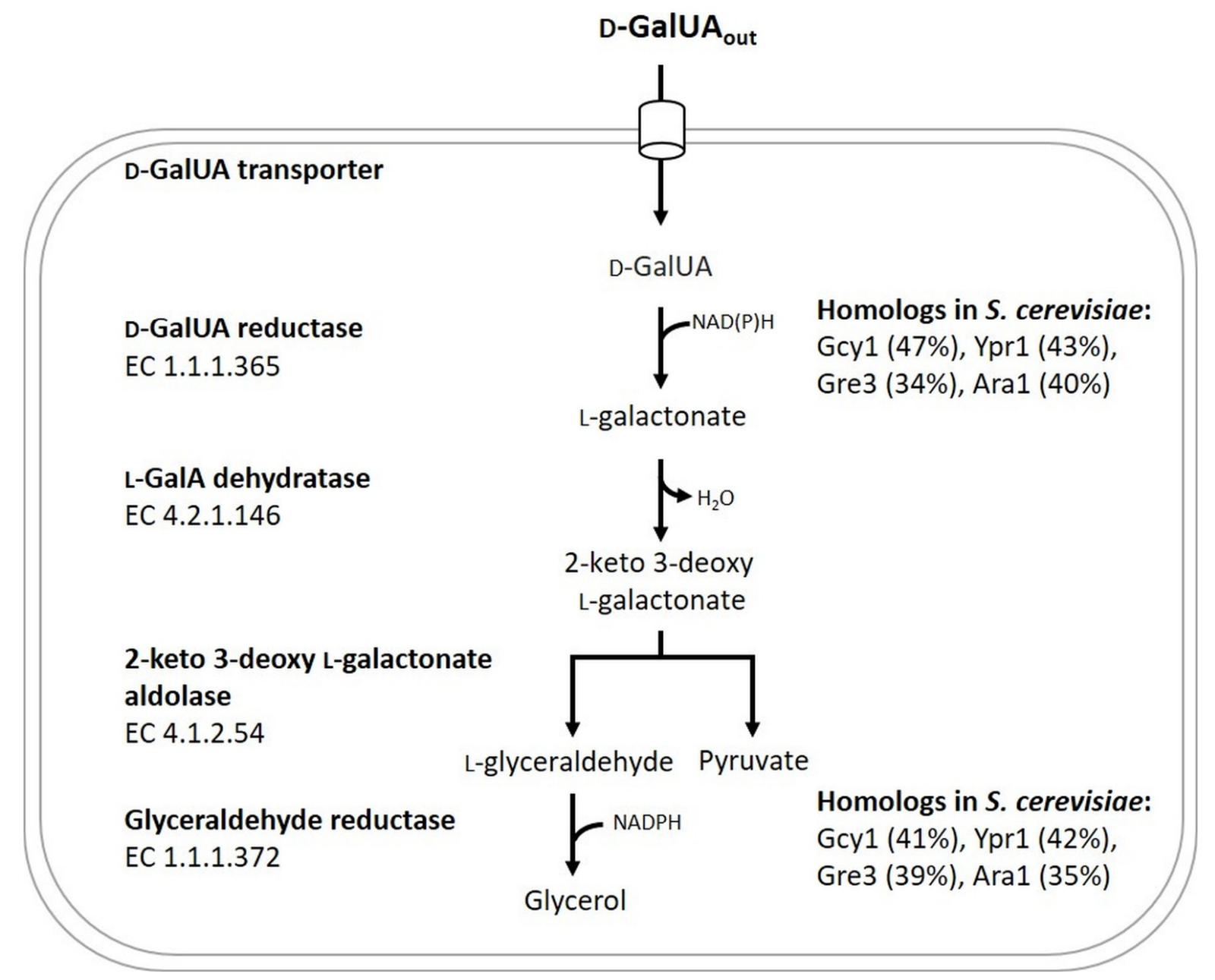

Figure 1. Overview of the enzymes comprising the D-galacturonic acid (D-GalUA) catabolic pathway commonly found in fungi able to utilize D-GalUA. In addition, S. cerevisiae proteins exhibiting a certain degree of homology to the fungal enzymes are shown. Percentage values in brackets indicate sequence identities between the respective yeast protein and the D-GalUA reductase Gar1 from T. reesei and the glyceraldehyde reductase Gld1 from T. reesei.

Among fungal organisms, baker's yeast became an attractive organism in the context of valorising hydrolysates of pectin-rich biomass [15] even though it is neither able to naturally metabolize D-GalUA nor L-arabinose. Baker's yeast is the organism of choice for the production of ethanol, and this has also been acknowledged in publications dealing with pectin-rich biomass $[2,15,16]$. Notably, the organism's popularity has profited from the ease of metabolic engineering. By virtue of S. cerevisiae's capacity for highly efficient homologous recombination, its accessibility to extensive targeted genetic modifications is extraordinary $[17,18]$. S. cerevisiae has already been successfully equipped with the ability to ferment pentose sugars to ethanol [19-22]. Notably, S. cerevisiae shows a relatively high tolerance to alcohols and organic acids, low $\mathrm{pH}$ and low oxygen levels [2,23-26]. These natural characteristics also contribute to the attractiveness of $S$. cerevisiae for valorising pectin-rich residues. 
There have already been several attempts to implement a heterologous D-GalUA pathway in S. cerevisiae $[11,27,28]$. However, the achieved rates of D-GalUA consumption were far from economically viable even under aerobic conditions and in the presence of a cosubstrate. Recently, our laboratory significantly improved the situation by constructing a S. cerevisiae strain able to consume D-GalUA with a specific rate of $0.23 \mathrm{~g} \mathrm{gCDW}^{-1} \mathrm{~h}^{-1}$ $(\mathrm{CDW}=$ cell dry weight [29]. A challenging aspect when considering homo-ethanol fermentation from pectin hydrolysates is the electron balance since D-GalUA is more oxidized than neutral sugars. In this context, we have considered glycerol an ideal co-substrate as a donor of electrons and embarked on the challenge to establish co-fermentation of D-GalUA and glycerol. In our study [29], a fungal D-GalUA pathway was inserted in a strain of the yeast S. cerevisiae previously equipped with an efficient NAD-dependent glycerol catabolic pathway. The study delivers the proof of concept for a co-fermentation of the two 'respiratory' carbon sources to ethanol and demonstrates a fast and complete consumption of D-GalUA in crude sugar beet pulp hydrolysate, at least under aerobic conditions [29].

It has been an interesting auxiliary result of the study conducted by Perpelea et al. [29] that the reference strain (without any heterologous D-GalUA transporter or catabolic enzyme) also consumed a certain amount of D-GalUA. It also became obvious that this conversion depended on the presence of the co-substrate glycerol. This result matched reports of other authors who previously showed that (i) some D-GalUA can be taken up by one or more endogenous uptake mechanism(s) in S. cerevisiae $[27,30]$ and (ii) one or more endogenous reductase(s) in S. cerevisiae can convert D-GalUA to L-GalA. This enzyme reaction corresponds to the first step of the fungal D-GalUA pathway [31,32]. The goal of the current study was to identify the enzyme(s) that is/are responsible for the observed conversion of D-GalUA to L-GalA in baker's yeast.

\section{Materials \& Methods}

\subsection{Strains, Medium Composition and General Cultivation Conditions}

All strains and plasmids used in this study are listed in Tables 1 and S1, respectively. Yeast cells were routinely grown on solid YPD medium containing $10 \mathrm{~g} \mathrm{~L}^{-1}$ yeast extract, $20 \mathrm{~g} \mathrm{~L}^{-1}$ peptone, $20 \mathrm{~g} \mathrm{~L}^{-1}$ glucose and $15 \mathrm{~g} \mathrm{~L}^{-1}$ agar. For yeast cultivation, the agar plates were placed in a static incubator at $30^{\circ} \mathrm{C}$. In case of selection of transformed strains, media were supplemented with $20 \mu \mathrm{L} \mathrm{mL}^{-1}$ phleomycin.

Table 1. S. cerevisiae strains used in this study.

\begin{tabular}{|c|c|c|}
\hline Strain & Genotype, Description & Reference \\
\hline CEN.PK113-1A & $M A T \alpha$ (prototrophic) & Euroscarf \\
\hline CEN.PK113-1A UBR2 ${ }_{C B S}$ & $M A T \alpha ; u b r 2:: U B R 2$ CBS 6412-13A & [33] \\
\hline CEN.PK RE & MAT $\alpha ; u b r 2:: U B R 2_{C B S} 6412-13 A ; g u t 1:: G U T 1_{J L 1}$ & This study * \\
\hline CEN.PK DHA & 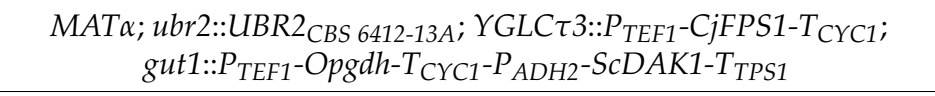 & [34] \\
\hline CEN.PK DHA gcy $1 \Delta$ & 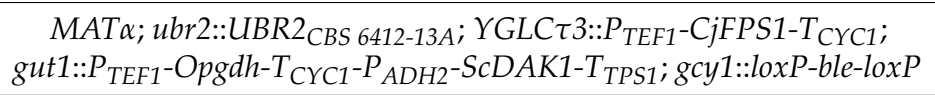 & This study \\
\hline CEN.PK DHA gre3s & 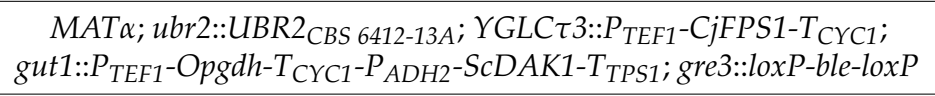 & This study \\
\hline CEN.PK DHA ypr1s & 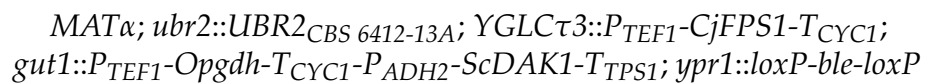 & This study \\
\hline
\end{tabular}

* The maximum specific growth rate of this strain has already been reported in the study of Ho et al. [35], where the strain was referred to as CEN.PK113-1A GUT1 ${ }_{L L 1}$ UBR2 $C B S$. The construction of this strain is described in Material and Methods of the current study. UBR2: encoding cytoplasmic ubiquitin-protein ligase; GUT1: encoding glycerol kinase; CjFPS1: encoding aquaglyceroporin from C. jadinii; Opgdh: encoding glycerol dehydrogenase from O. parapolymorpha; DAK1: encoding dihydroxyacetone kinase; GCY1, YPR1 and GRE3: encoding unspecific aldo-keto reductases. 
E. coli $\mathrm{DH} 5 \alpha$ was used for plasmid maintenance and isolation. E. coli strains carrying plasmids were routinely grown at $250 \mathrm{rpm}$ and $37^{\circ} \mathrm{C}$ in lysogeny broth containing $10 \mathrm{~g} \mathrm{~L}^{-1}$ peptone, $5 \mathrm{~g} \mathrm{~L}^{-1}$ yeast extract, $10 \mathrm{~g} \mathrm{~L}^{-1} \mathrm{NaCl}\left(\mathrm{pH}\right.$ 7.0) and $100 \mathrm{mg} \mathrm{L}^{-1}$ ampicillin for selection. Plasmids were isolated by using the GeneJET ${ }^{\mathrm{TM}}$ Plasmid Miniprep Kit (Thermo Fisher Scientific, Waltham, MA, USA).

\subsection{General Molecular Biology Techniques}

Preparative PCRs for cloning were performed using Phusion ${ }^{\circledR}$ High-Fidelity DNA Polymerase (New England Biolabs, Frankfurt am Main, Germany). PCR conditions were adapted according to the guidelines of the manufacturer. PCR products were purified using the GeneJET ${ }^{\mathrm{TM}}$ PCR Purification Kit (Thermo Fisher Scientific). Transformation of $S$. cerevisiae was performed according to the lithium acetate method described by Gietz et al. [36].

\subsection{Construction and Verification of Deletion Strains}

For gene disruption, deletion cassettes containing the phleomycin (ble) resistance marker were amplified from pUG66 [37] using the primers listed in Table S2 (Supplementary Material). The primers used for amplification contained at their $5^{\prime}$-terminus a $40-50 \mathrm{bp}$ sequence complementary to the region immediately upstream or downstream of the start or stop codon of the gene to be deleted. The cassettes were used to transform S. cerevisiae and their integration at the target locus by homologous recombination resulted in deletion of the respective genes. Proof of the correct integration of all deletion cassettes was performed by diagnostic PCR (primers listed in Table S2, Supplementary Material) using OneTaq Quick-Load DNA Polymerase (New England Biolabs) according to the manufacturer's guideline. Single cell colonies obtained after transformation were re-streaked on selective agar plates. Genomic DNA was isolated according to a modified protocol from Hoffman and Winston [38]. Approximately $50 \mathrm{mg}$ of cells was suspended in $200 \mu \mathrm{L}$ of TE buffer (10 mM Tris, $1 \mathrm{mM}$ EDTA, pH 8.0). Afterwards, $300 \mathrm{mg}$ of acid-washed glass beads (diameter of $0.425-0.6 \mathrm{~mm}$ ) and $200 \mu \mathrm{L}$ of phenol/chloroform/isoamyl alcohol (25:24:1) were added. The tubes were vortexed at a maximum speed for $2 \mathrm{~min}$ and centrifuged at $15,700 \mathrm{~g}$ for $10 \mathrm{~min}$. From the aqueous phase, $1 \mu \mathrm{L}$ was used as template in $20 \mu \mathrm{L}$ PCR reactions.

\subsection{Construction and Verification of the Reverse-Engineered Strain CEN.PK RE}

The reverse-engineered strain CEN.PK RE is a derivate of the previously described strain CEN.PK113-1A UBR2 $C B S$ [33]. To construct the strain CEN.PK RE (Table 1), the endogenous GUT1 allele of strain CEN.PK113-1A UBR2 ${ }_{C B S}$ was replaced by the GUT1 allele from strain JL1 [35]. The only difference was a single point mutation within the GUT1 expression cassette, as reported by Ho et al. [35]. This point mutation improves growth in synthetic glycerol medium. The allele replacement was achieved by using the same two-step GIN11 counter-selectable strategy and primers described by Ho et al. [35].

\subsection{Media for Analysing Glycerol and D-GalUA Utilization of Engineered S. cerevisiae Strains}

All pre-cultures were cultured in synthetic medium containing $20 \mathrm{~g} \mathrm{~L}^{-1}$ glucose and ammonium sulphate as carbon and nitrogen source, respectively. All experiments for assessing glycerol and D-GalUA consumption in shake flask batch cultivation were performed in synthetic medium, containing $60 \mathrm{~mL} \mathrm{~L}^{-1}\left(75.6 \mathrm{~g} \mathrm{~L}^{-1}\right)$ glycerol and $5 \mathrm{~g} \mathrm{~L}^{-1}$ D-GalUA (D-galacturonic acid sodium salt, Chemodex, St. Gallen, Switzerland) as carbon sources and urea as nitrogen source. The synthetic medium was prepared according to Verduyn et al. [39] and contained $3 \mathrm{~g} \mathrm{~L}^{-1} \mathrm{KH}_{2} \mathrm{PO}_{4}, 0.5 \mathrm{~g} \mathrm{~L}^{-1} \mathrm{MgSO}_{4} \cdot 7 \mathrm{H}_{2} \mathrm{O}, 15 \mathrm{mg} \mathrm{L}-1$ EDTA, $4.5 \mathrm{mg} \mathrm{L}^{-1} \mathrm{ZnSO}_{4} \cdot 7 \mathrm{H}_{2} \mathrm{O}, 0.84 \mathrm{mg} \mathrm{L}^{-1} \mathrm{MnCl}_{2} \cdot 2 \mathrm{H}_{2} \mathrm{O}, 0.3 \mathrm{mg} \mathrm{L}-1 \mathrm{CoCl}_{2} \cdot 6 \mathrm{H}_{2} \mathrm{O}$, $0.3 \mathrm{mg} \mathrm{L}^{-1} \mathrm{CuSO}_{4} \cdot 5 \mathrm{H}_{2} \mathrm{O}, 0.4 \mathrm{mg} \mathrm{L}{ }^{-1} \mathrm{NaMoO}_{4} \cdot 2 \mathrm{H}_{2} \mathrm{O}, 4.5 \mathrm{mg} \mathrm{L}^{-1} \mathrm{CaCl}_{2} \cdot 2 \mathrm{H}_{2} \mathrm{O}, 3 \mathrm{mg} \mathrm{L}^{-1}$ $\mathrm{FeSO}_{4} \cdot 7 \mathrm{H}_{2} \mathrm{O}, 1 \mathrm{mg} \mathrm{L}^{-1} \mathrm{H}_{3} \mathrm{BO}_{3}$ and $0.1 \mathrm{mg} \mathrm{L}^{-1} \mathrm{KI}$. After heat sterilization of the salts, $1 \mathrm{~mL} \mathrm{~L}^{-1}$ of a vitamin stock solution was added, resulting in the following final con- 
centrations: $0.05 \mathrm{mg} \mathrm{L}^{-1} \mathrm{D}-(+)$-biotin, $1 \mathrm{mg} \mathrm{L}^{-1}$ D-pantothenic acid hemicalcium salt, $1 \mathrm{mg} \mathrm{L}^{-1}$ nicotinic acid, $25 \mathrm{mg} \mathrm{L}^{-1}$ myo-inositol, $1 \mathrm{mg} \mathrm{L}^{-1}$ thiamine chloride hydrochloride, $1 \mathrm{mg} \mathrm{L}^{-1}$ pyridoxine hydrochloride and $0.2 \mathrm{mg} \mathrm{L}^{-1} 4$-aminobenzoic acid. In case urea was used as the nitrogen source (in main culture media), an appropriate aliquot of a stock solution was added after autoclaving to obtain a final concentration of $2.8 \mathrm{~g} \mathrm{~L}^{-1}$, while in pre-cultures, $5 \mathrm{~g} \mathrm{~L}^{-1}$ ammonium sulphate was added before heat sterilization. The $\mathrm{pH}$ was adjusted to 6.5 with $4 \mathrm{M} \mathrm{KOH}$ for the synthetic glucose medium. The $\mathrm{pH}$ of the synthetic glycerol medium containing D-GalUA was adjusted to either 3.0 using $2 \mathrm{M} \mathrm{H}_{3} \mathrm{PO}_{4}$ or 5.0 using $4 \mathrm{M} \mathrm{KOH}$.

\subsection{Characterization of S. cerevisiae in Shake Flask Batch Cultivations}

Cells from a single colony were used to inoculate $3 \mathrm{~mL}$ of synthetic glucose medium in a $10 \mathrm{~mL}$ glass tube and were incubated at orbital shaking of $200 \mathrm{rpm}$ and $30^{\circ} \mathrm{C}$ for $16 \mathrm{~h}$. The pre-culture was used to inoculate $10 \mathrm{~mL}$ of the same medium in a $100 \mathrm{~mL}$ Erlenmeyer flask, adjusting an $\mathrm{OD}_{600}$ of 0.2 . This culture, hereafter referred to as intermediate culture, was cultivated under the same conditions for $24 \mathrm{~h}$. The appropriate culture volume from the intermediate culture (in order to later adjust an $\mathrm{OD}_{600}$ of 0.2 in $50 \mathrm{~mL}$ or $100 \mathrm{~mL}$ of synthetic glycerol medium containing D-GalUA) was centrifuged at $800 \mathrm{~g}$ for $5 \mathrm{~min}$. The cell pellet was then washed once by re-suspending the cells in synthetic glycerol medium containing D-GalUA. The cell suspension was centrifuged again and re-suspended in $50 \mathrm{~mL}$ or $100 \mathrm{~mL}$ of the same medium in a $500 \mathrm{~mL}$ Erlenmeyer flask. The main cultures were incubated at orbital shaking of $200 \mathrm{rpm}$ and $30^{\circ} \mathrm{C}$, and samples for $\mathrm{OD}_{600}$ determination and HPLC analysis were taken at regular time intervals.

\subsection{Metabolite Analysis by HPLC}

The samples (1.0 mL culture supernatant) were filtered through $0.2 \mu \mathrm{m}$ Minisart RC membrane filters (Sartorius, Göttingen, Germany) and stored at $-20{ }^{\circ} \mathrm{C}$ until analysis. Detection and quantification of glycerol and D-GalUA was performed using a Waters HPLC system (Eschborn, Germany) consisting of a binary pump (Waters 1525), an injector system (Waters 2707), a Waters column heater module WAT038040 and a refractive index detector (Waters 2414). An Aminex HPX-87H cation exchange column (Bio-Rad, München, Germany) coupled to a Micro-guard ${ }^{\circledR}$ cation exchange column (Bio-Rad) was used for chromatography. As an eluent, $5 \mathrm{mM} \mathrm{H}_{2} \mathrm{SO}_{4}$ at a flow rate of $0.6 \mathrm{~mL} \mathrm{~min}^{-1}$ was used. The column was kept at $45^{\circ} \mathrm{C}$. A sample volume of $20 \mu \mathrm{L}$ was injected. Under these conditions, the retention times were about $14 \mathrm{~min}$ for glycerol and $8.5 \mathrm{~min}$ for D-GalUA. L-GalA was detected under the same conditions. Notably, L-GalA has not been commercially available and the L-GalA used here as a standard was obtained by hydrolysis of L-galactono1,4-lactone (Sigma-Aldrich, Schnelldorf, Germany) (titrating the solution to $\mathrm{pH} 8$ with $\mathrm{NaOH}$ ) and was kindly provided by Peter Richard (VTT, Espoo, Finland). This self-made standard resulted in two peaks with retention times of 9.3 and $9.7 \mathrm{~min}$. Data were analysed using the Breeze 2 software (Waters).

\subsection{Selectable Genetic Marker Replacement in Multicopy Plasmids for GCY1 Overexpression}

The construction of a $2 \mu$-based plasmid p424GCY1 for the overexpression of GCY1 has been described in Nguyen and Nevoigt [40]. This plasmid and the corresponding empty vector $\mathrm{p} 424 \mathrm{GPD}$ [41] contain the TRP1 gene as the selectable genetic marker. The auxotrophic marker was replaced by the dominant marker conferring resistance to phleomycin in order to be used in prototrophic S. cerevisiae strains. The ble (from bacterial transposon Tn5) cassette was amplified from pUG66 [36] using primers listed in Table S3 (Supplementary Material). The PCR product and the respective plasmid were used to transform S. cerevisiae, and the marker replacement occurred via in vivo recombination. The correct marker replacement was checked via diagnostic PCR using the primers listed in Table S3 (Supplementary Material) and OneTaq Quick-Load DNA Poly- 
merase (New England Biolabs) according to the manufacturer's guideline. The correct transformants were used for the D-GalUA reductase enzyme assay.

\subsection{In Vitro Measurement of D-GalUA Reductase Activity}

Cell disruption and enzyme assay were carried out following the procedure described by Biz et al. [11] with slight modifications. Yeast cells were grown overnight in $50 \mathrm{~mL}$ liquid YPD medium supplemented with $30 \mu \mathrm{g} / \mathrm{mL}$ of the antibiotic phleomycin. A culture volume of $50 \mathrm{~mL}$ with an $\mathrm{OD}_{600}$ of 5 (corresponding to ca. $120 \mathrm{mg}$ CDW) was harvested. To ensure harvesting of the same number of cells in cultures of different optical densities, the appropriate culture volume was calculated based on the actual $\mathrm{OD}_{600}$ of the culture. Cells were washed once with $50 \mathrm{~mL}$ water and re-suspended in $1 \mathrm{~mL} 50 \mathrm{mM}$ sodium phosphate buffer ( $\mathrm{pH} 7$ ) with addition of the EDTA-free Protease inhibitor cocktail cOmplete (Roche, Basel, Switzerland) according to the manufacturer's guideline, and $0.5 \mathrm{~g}$ glass beads ( $0.4 \mathrm{~mm}$ diameter) was added. Cells were disrupted by vigorous vortexing for 10 min at $4{ }^{\circ} \mathrm{C}$ using Genie 2 Vortex Mixer (Scientific Industries, New York, USA) equipped with an adapter holding for $1.5 \mathrm{~mL}$ tubes. The samples were centrifuged for $15 \mathrm{~min}$ at $16000 \mathrm{~g}$ and $4{ }^{\circ} \mathrm{C}$ to remove glass beads and cell debris. The enzyme activity was assayed in the supernatant by recording the decrease in absorbance at $340 \mathrm{~nm}$ caused by the oxidation of NADPH in a mixture containing $100 \mathrm{mM}$ sodium phosphate buffer ( $\mathrm{pH} 7), 0.2 \mathrm{mM}$ $\mathrm{NADPH}, 10 \mathrm{mM}$ D-GalUA, and $50 \mu \mathrm{L}$ crude yeast extract in a total volume of $1 \mathrm{~mL}$. After incubating the mixture without D-GalUA at $30{ }^{\circ} \mathrm{C}$ for $1 \mathrm{~min}$, the reaction was started by the addition of $100 \mu \mathrm{L}$ of $100 \mathrm{mM}$ D-GalUA. Protein concentration in crude cell extracts was determined by the BCA assay [42] using a kit from Thermo Scientific.

\section{Results}

3.1. The S. cerevisiae Derivate CEN.PK113-1A Equipped with the NAD-Dependent DHA Pathway for Glycerol Utilization Was Able to Convert D-GalUA to L-GalA

As mentioned in the introduction, a glycerol-utilizing derivative of the well-characterized S. cerevisiae strain CEN.PK consumed some D-GalUA when added to synthetic glycerol medium [29]. In the respective strain referred to here as CEN.PK DHA [34], the endogenous so-called L-glycerol-3-phosphate (L-G3P) pathway for glycerol catabolism (reviewed in Klein et al. [43]) was replaced by the NAD-dependent dihydroxyacetone (DHA) pathway [44]. Moreover, the strain carried an expression cassette for a heterologous aquaglyceroporin from Cyberlindnera jadinii for improved glycerol uptake [45] and a replacement of the native UBR2 allele by the respective allele from the natural $S$. cerevisiae isolate CBS 6412-13A [33]. The UBR2 gene encodes a cytoplasmic ubiquitin-protein ligase identified in the study of Swinnen et al. [33]. The authors focused on a QTL analysis to identify the crucial mutations allowing the natural isolate CBS 6412-13A to grow in synthetic glycerol medium in contrast to the popular laboratory strain CEN.PK113-1A. One of the crucial genetic determinants was the sequence of the UBR2 gene. Swinnen et al. [33] demonstrated that the endogenous UBR2 allele of CEN.PK113-1A encodes for a truncated version of the Ubr2 protein. Moreover, the authors demonstrated that the replacement of the endogenous UBR2 allele by the allele from strain CBS 6412-13A is essential for establishing growth in synthetic glycerol medium in CEN.PK strains. The combination of the above-mentioned modifications in the strain CEN.PK DHA allow the strain to grow in synthetic glycerol medium with a maximum specific growth rate of $0.24 \mathrm{~h}^{-1}$ [34] while wild-type CEN.PK strains are unable to grow at all under these conditions [46].

The finding of Perpelea et al. [29] that the strain CEN.PK DHA (Gly reference strain) utilizes some D-GalUA has been obtained in shake flask experiments conducted with an initial $\mathrm{pH}$ of 5 . Here, we repeated the experiment at an initial $\mathrm{pH}$ of 3 since $S$. cerevisiae strains (without a dedicated D-GalUA transporter) have previously been reported to show a significantly faster natural uptake of $\mathrm{D}-\mathrm{GalUA}$ at $\mathrm{pH}$ values below 3.51, which is the $\mathrm{pK}_{\mathrm{a}}$ of D-GalUA [30]. A comparison of D-GalUA consumption of the CEN.PK DHA strain at initial $\mathrm{pH}$ values of 3 and 5 confirmed that D-GalUA consumption increased significantly at 
the lower $\mathrm{pH}$ (Figure 2). As a next step, we checked culture supernatants for the presence of L-GalA since Benz et al. [31] reported that a S. cerevisiae strain expressing a heterologous D-GalUA transporter (but no heterologous enzymes for D-GalUA catabolism) converted D-GalUA to L-GalA. Indeed, HPLC analysis of the culture supernatants obtained with our strain in the presence of D-GalUA revealed a decrease in the D-GalUA peak area. Moreover, the size of a peak with a retention time of $\sim 9.7 \mathrm{~min}$ that was not visible in a control cultivation in synthetic glycerol medium without the addition of D-GalUA increased over time. We assume that the peak corresponds to L-GalA (Material and Methods). We used LC-MS to verify the presence of L-GalA in the supernatants of the CEN.PK DHA culture grown for $144 \mathrm{~h}$ in synthetic glycerol medium supplemented with D-GalUA (initial $\mathrm{pH} 3$ ).

A

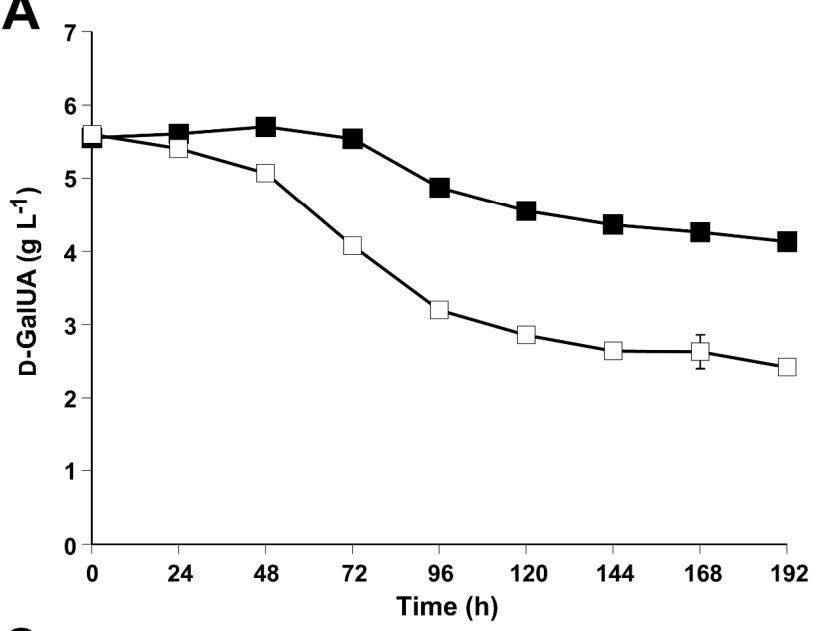

C

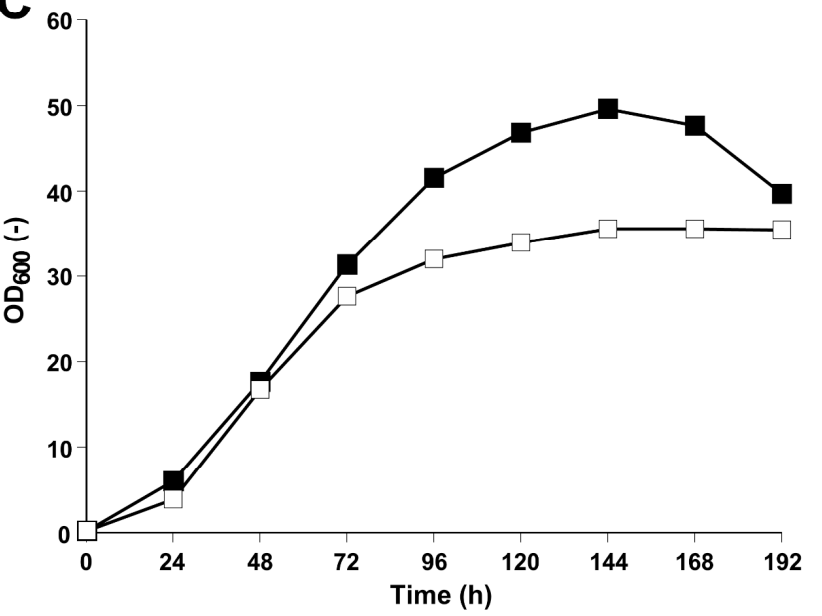

B

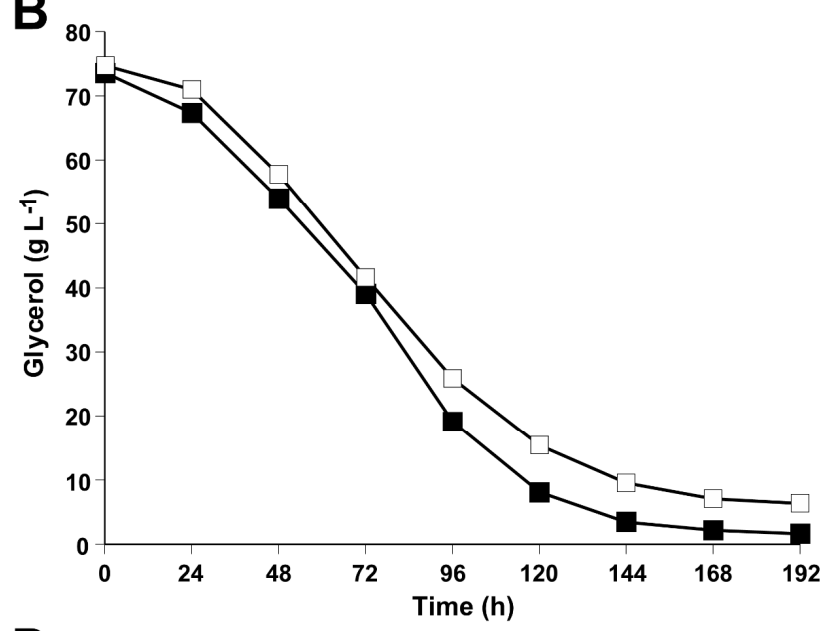

D

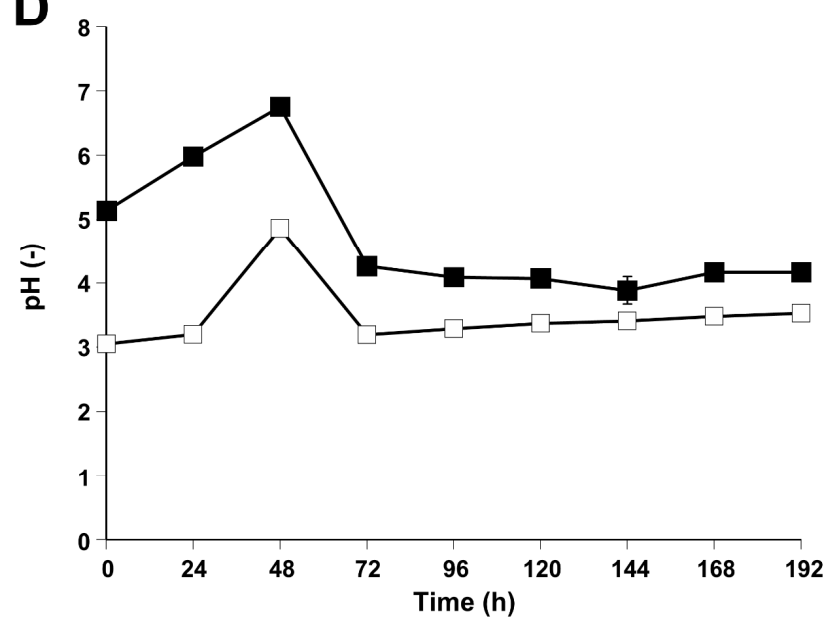

$\mathrm{pH} 5-\llcorner\mathrm{pH} 3$

Figure 2. D-GalUA consumption profile of the strain CEN.PK DHA in synthetic glycerol medium supplemented with D-GalUA at an initial pH of 5 and 3. Cultivations were performed in $500 \mathrm{~mL}$ flasks containing $100 \mathrm{~mL}$ of synthetic medium with glycerol and D-GalUA with an initial pH of 5 (closed squares) or an initial pH of 3 (open squares) and urea as the source of nitrogen. Samples were taken in $24 \mathrm{~h}$ intervals, and culture supernatants were analysed by HPLC to follow the consumption of D-GalUA (A) and glycerol (B). Growth was recorded by determining the optical density of the cultures at a wavelength of $600 \mathrm{~nm}\left(\mathrm{OD}_{600}\right)(\mathrm{C})$. $\mathrm{pH}$ variation was measured over time (D). All mean values and standard deviations were derived from biological triplicates. 


\subsection{Deletion of GCY1 Abolished Formation of L-GalA from D-GalUA}

In order to identify candidate genes encoding the endogenous D-GalUA reducing activity in S. cerevisiae, we first searched for sequence similarities among baker's yeast proteins to the best-known fungal D-GalUA reductases, i.e., GaaA from Aspergillus niger (NCBI Accession number: ABQ53587.1) [47] and Gar1 from T. reesei (NCBI Accession number: AAX54673.1) [48], by performing a BLAST (basic local alignment search tool) analysis. While this search did not reveal any yeast ortholog for A. niger GaaA, we identified the four proteins Gcy1 (NCBI Accession number: NP_014763.1), Ypr1 (NCBI Accession number: NP_010656.1), Gre3 (NCBI Accession number: NP_011972.1) and Ara1 (NCBI Accession number: NP_009707.3) from S. cerevisiae which show relatively high sequence identities to the T. reesei Gar1 (47\%, 43\%, 34\% and 40\%, respectively) (Figure 1). These proteins were previously characterized as aldo-keto reductases with a wide substrate spectrum [49,50]. Notably, we did not further analyse Ara1 since it prefers to catalyse the reaction in the direction of aldose oxidation, as shown by Kim et al. [51].

To test the contribution of the three identified S. cerevisiae aldo-keto reductases in the conversion of D-GalUA to L-GalA in the strain CEN.PK DHA, single gene deletions of GCY1, YPR1 and GRE3 were constructed in this genetic background. The resulting deletion mutants were characterized in synthetic glycerol medium supplemented with D-GalUA. In accordance with the previous findings regarding natural D-GalUA uptake by S. cerevisiae, the initial $\mathrm{pH}$ of the medium was set to 3 . The D-GalUA concentration in the cultivations of the ypr 1 and gre3 deletion strains decreased with the same kinetics as observed for the reference strain (CEN.PK DHA). However, D-GalUA conversion was significantly reduced for the gcy $1 \Delta$ mutant (Figure 3). In contrast, growth and glycerol utilization of all mutants remained unaffected. The resulting hypothesis that Gcy1 is the endogenous enzyme that converts D-GalUA to L-GalA was substantiated by the fact that no L-GalA peak was detectable in HPLC chromatograms of supernatants taken from the culture of the gcy $1 \Delta$ mutant throughout the entire cultivation (Figure 4). The absence of the L-GalA peak in this strain was also confirmed for the culture taken after $144 \mathrm{~h}$ of cultivation via LC-MS. In contrast, a peak with increasing area for L-GalA was visible in the culture supernatants of the reference strain, the ypr1 and the gre3 deletion mutant (Figure 4). Interestingly, all HPLC chromatograms from samples taken after $144 \mathrm{~h}$ of cultivation revealed a peak of unknown identity (retention time $\sim 7.7 \mathrm{~min}$ ) whose area was significantly increased in the gcy $1 \Delta$ mutant compared with all other strains (Figure 4). The appearance of the respective peak was dependent on the presence of D-GalUA in the medium. This was confirmed by cultivating the gcy $1 \Delta$ mutant in the same synthetic glycerol medium but without D-GalUA.

\subsection{Conversion of D-GalUA to L-GalA Was Not Dependent on the DHA Pathway for Glycerol Catabolism}

GCY1 has, in several independent studies, been demonstrated to encode a strictly NADPH-dependent enzyme [50,52-54]. We scrutinized whether Gcy1 merely provided the NADPH for the in vivo D-GalUA reduction while the actual reduction of D-GalUA was carried out by another endogenous enzyme. In this context, it becomes relevant to note that the S. cerevisiae strain CEN.PK DHA (used in the above experiments to delete GCY1, $Y P R 1$ and GRE3, respectively) carries multiple genetic modifications, including the strong overexpression of Opgdh, which is a dehydrogenase (Figure S1B). These modifications allow the superior growth of this strain in synthetic glycerol medium. Although we considered it unlikely that the heterologous Opgdh in the respective strain would have a side activity towards reduction of D-GalUA, we decided to check whether the D-GalUA-reducing activity detected in the presence of glycerol in the strain CEN.PK DHA was dependent on the DHA pathway. Unfortunately, we could not use the corresponding wild-type CEN.PK113-1A strain (Figure S1A) since this strain cannot grow in synthetic glycerol medium at all [46] and the in vivo D-GalUA reducing activity can only be observed during co-consumption of glycerol. A solution was the use of a reverse-engineered derivative of the strain CEN.PK 113-1A. This strain—referred to here as CEN.PK RE (Table 1)—does not contain the DHA 
pathway but can still grow in synthetic glycerol medium with a moderate rate solely based on the L-G3P pathway (Figure S1C). The only genetic modifications in strain CEN.PK RE compared with the wild-type CEN.PK113-1A are the following allele replacements: (i) the endogenous UBR2 allele by UBR2 from strain CBS 6412-13A and (ii) the wild-type CEN.PK GUT1 allele by the GUT1 allele from JL1 [35]. When the strain CEN.PK RE was tested in synthetic glycerol medium supplemented with D-GalUA at an initial pH 3, it did not consume any D-GalUA before $144 \mathrm{~h}$ of cultivation (Supplementary Material, Figure S2A). It is assumed that this delay matches the significantly slower biomass formation accompanied by a reduced consumption of glycerol (compared with the strain CEN.PK DHA) (Supplementary Material, Figure S2B,C). Still, the HPLC chromatogram of the medium sample taken after $192 \mathrm{~h}$ of cultivation displayed a small L-GalA peak (Supplementary Material, Figure S2D). The low quantity fits to the comparatively low amount of D-GalUA consumed (Supplementary Material, Figure S2A). Thus, the results suggest that the existence of the endogenous D-GalUA reductase activity in S. cerevisiae is independent of the genetic modifications in connection to the DHA pathway. However, the more efficient glycerol consumption in the DHA pathway strain seemed to provide the required reducing power faster, thereby facilitating the conversion.

A

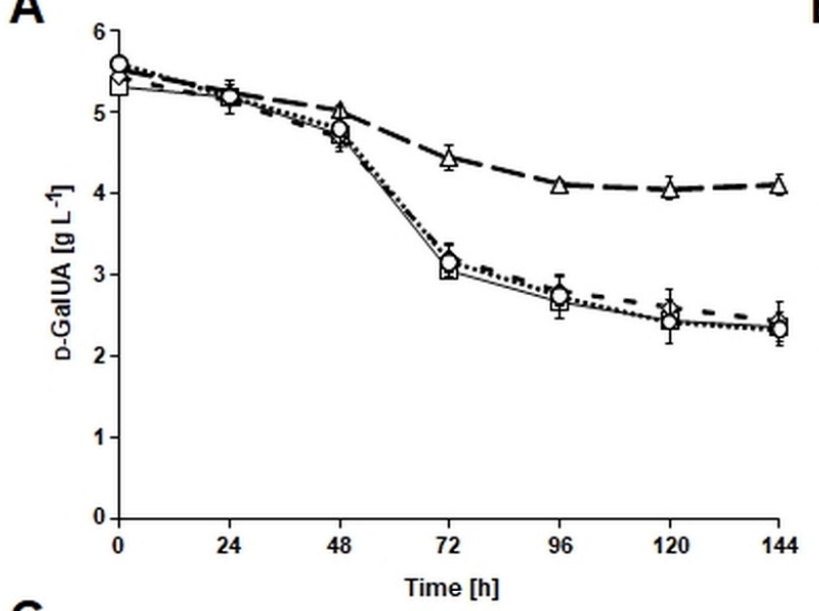

C

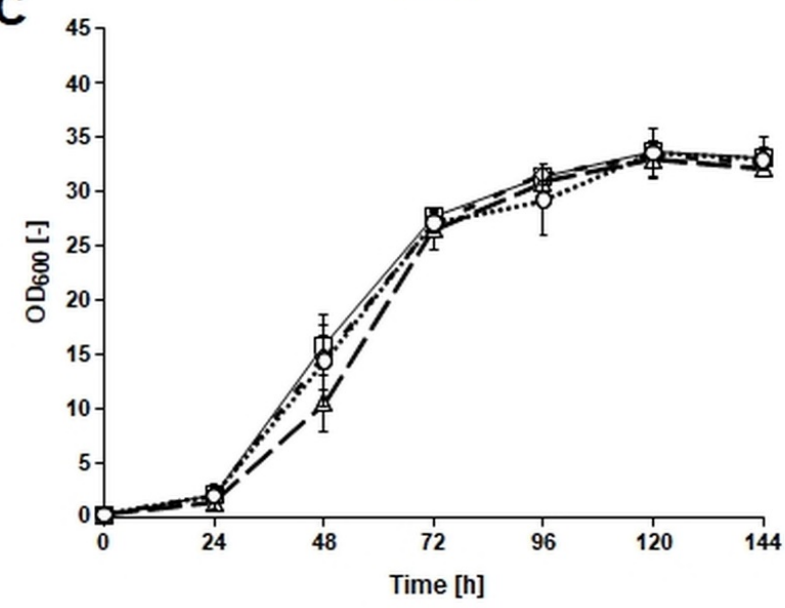

B

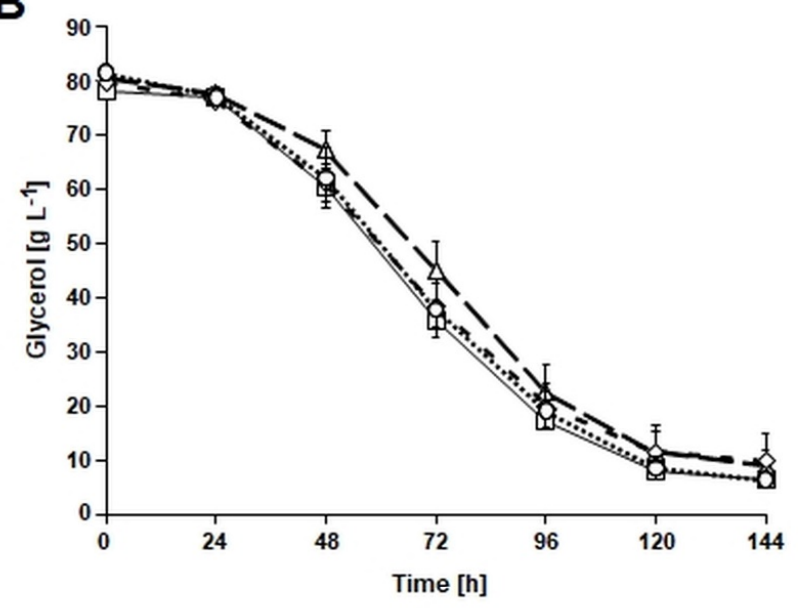

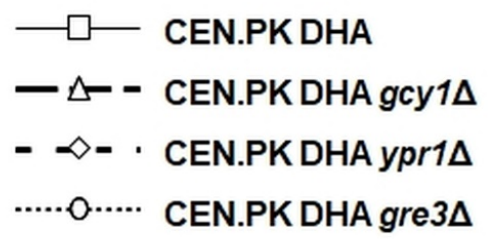

Figure 3. D-GalUA and glycerol utilization, as well as biomass formation of the strain CEN.PK DHA, and the impact of deleting the genes (GCY1, YPR1 and GRE3) encoding unspecific aldo-keto reductases and showing homology to fungal enzymes involved in the D-GalUA catabolic pathway. Strains were cultivated in synthetic glycerol medium supplemented with D-GalUA and urea as the source of nitrogen. Cultivations were performed in $500 \mathrm{~mL}$ flasks containing $50 \mathrm{~mL}$ medium with an initial $\mathrm{pH}$ of 3. Samples were taken in $24 \mathrm{~h}$ intervals, and culture supernatants were analysed by HPLC to follow the consumption of D-GalUA (A) and glycerol (B). Growth was recorded by determining the optical density of the cultures at a wavelength of $600 \mathrm{~nm}\left(\mathrm{OD}_{600}\right)(\mathrm{C})$. All mean values and standard deviations were derived from biological triplicates. 

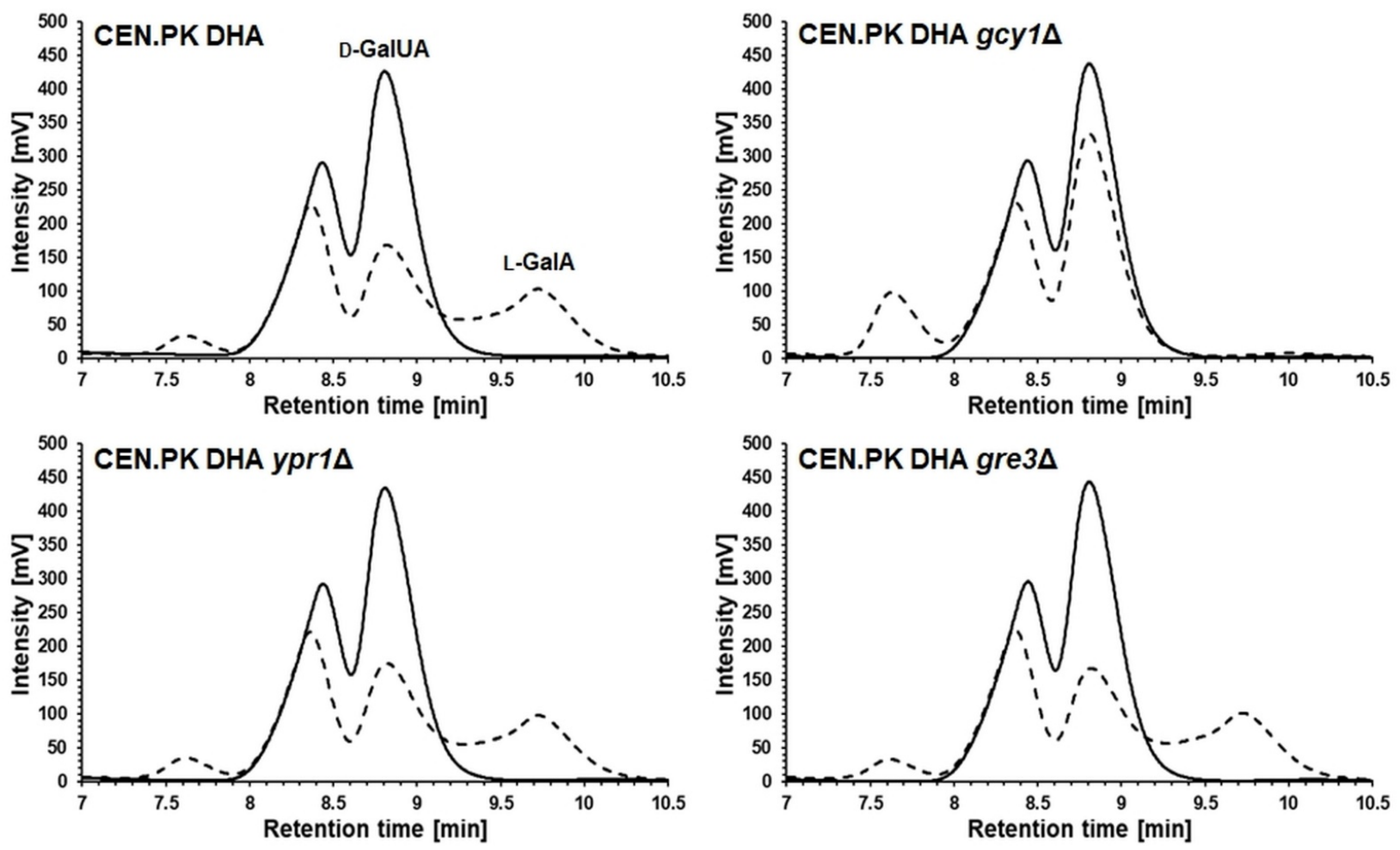

Figure 4. HPLC chromatograms of culture supernatants from shake flask cultivations in synthetic glycerol medium supplemented with D-GalUA conducted with the reference strain CEN.PK DHA and the three isogenic mutant strains in which GCY1, GRE3 or YPR1 (encoding unspecific aldo-keto reductases) was deleted. Cultivation conditions are described in the figure caption of Figure 3. D-GalUA and L-GalA were detected via the Waters 2412 RI detector. The retention time of D-GalUA was $\sim 8.8 \mathrm{~min}$, and the retention time of L-GalA was $\sim 9.7 \mathrm{~min}$. For each strain, a sample after $144 \mathrm{~h}$ (dashed line) was compared with a sample from the respective strain that was taken at the beginning of the batch cultivation in shake flasks (straight line).

\subsection{Overexpression of GCY1 Resulted in Increased In Vitro Conversion of D-GalUA}

In order to verify that Gcy1 is directly responsible for the conversion of D-GalUA to L-GalA, we transformed the strains CEN.PK DHA and CEN.PK113-1A with a $2 \mu$-based multicopy vector overexpressing GCY1 (p424GCY1-ble, Table S1). The same two strains were transformed with the empty vector (p424GPD-ble, Table S1) to obtain the respective isogenic reference strains without GCY1 overexpression. The in vitro measurement of D-GalUA reducing activity with NADPH as a co-factor detected a specific enzyme activity of $294 \pm 44 \mathrm{mU} / \mathrm{mg}$ protein in crude cell extracts of the two GCY1 overexpressing strains. In contrast, the negative controls with the empty vector did not reveal any enzyme activity under these conditions. Moreover, both strains did not show any D-GalUA reductase activity if NADH was used as co-factor. The results confirm that Gcy1 is able to reduce D-GalUA in an NADPH-dependent manner.

\section{Discussion}

In this study, we provide the experimental evidence that the aldo-keto reductase Gcy1 is mainly responsible for the slight enzymatic conversion of D-GalUA to L-GalA that has been observed when our $S$. cerevisiae strain solely equipped with the DHA pathway for glycerol catabolism was cultivated in a mixture of glycerol and D-GalUA [29]. Apart from the respective results obtained in our previous work (at initial pH 5) and in our current study (at initial pH of 3), several past reports of other authors also pointed to the ability of $S$. cerevisiae to take up D-GalUA and/or convert it to L-GalA. For example, L-GalA was intracellularly detected by means of LC-MS/MS in a strain solely carrying a heterologous transporter for D-GalUA uptake [31]. In another study, the authors expressed 
a D-GalUA reductase from Cryptococcus diffluens in S. cerevisiae in order to demonstrate the production of L-GalA from D-GalUA [32]. The respective reference strain (i.e., wild-type $S$. cerevisiae without any heterologous reductase) also produced a small amount of L-GalA from D-GalUA if galactose was used as co-substrate. In addition to the fact that $S$. cerevisiae exhibits a D-GalUA reducing activity, our current study also confirms that this organism can take up D-GalUA to a certain degree even at $\mathrm{pH}$ values higher than the $\mathrm{pK}_{\mathrm{a}}$ of $\mathrm{D}-\mathrm{GalUA}$ (3.51) [30]. Nevertheless, the observed D-GalUA consumption (related to biomass) detected in the current study was faster in the experiment conducted at the initial $\mathrm{pH}$ of 3 , a result matching the assumption of a more efficient D-GalUA uptake at lower pH (Figure 2).

Based on the data generated in the study of Perpelea et al. [29], we know that D-GalAU is only consumed by the strain CEN.PK DHA if the co-substrate glycerol is added, which allows growth. It also seems that the enzymatic conversion of D-GalAU to L-GalA mainly occurs during the phase of the cultivation in which growth is most pronounced (Figure 3). Gcy1 has been shown to be strictly dependent on NADPH, and it is obvious that the redox equivalents have to be delivered during the metabolism of glycerol. It is likely that the NADPH is mainly formed during the oxidative pentose phosphate pathway and that the flux through this pathway is increased during growth [55].

Gcy1 was initially identified as a galactose-inducible crystalline-like protein (yeast homolog of eye-lens protein $\rho$-crystallin from European common frog) [56]. Because of its sequence identity to a peptide fragment of glycerol dehydrogenase from $A$. niger, it was originally speculated that Gcy1 might function as a GDH converting glycerol to DHA since Gcy1 expression was strongly increased upon osmo-stress [57,58]. In addition, the expression of GCY1 was significantly higher in medium containing glycerol as the sole source of carbon, but not ethanol [59]. However, all efforts to measure GDH activity in vitro have failed so far $[40,57]$. Nonetheless, Zhang et al. [60] claimed an improved glycerol utilization in a S. cerevisiae strain in which the genes GCY1 (encoding an NADPH-dependent aldo-keto reductase) and DAK1 (encoding a dihydroxyacetone kinase) are overexpressed.

Gcy1 seems to be a relatively promiscuous enzyme with a broad substrate spectrum. Chang et al. [50] expressed the GCY1 gene from S. cerevisiae in E. coli, and the activity of the gene product was tested with several substrates such as D,L-glyceraldehyde, $\rho$-nitrobenzaldehyde, benzaldehyde and phenylglyoxal. D,L-glyceraldehyde was the substrate with the highest $\mathrm{k}_{\mathrm{cat}}$, and NADPH was shown to be the co-factor. Interestingly, S. cerevisiae Gcy1 shows high homology to different enzymes contributing to D-GalUA catabolism in filamentous fungi. It shares a high sequence homology with Gar1 from T. reesei (47\%), A. niger [61] (51\%) and Botrytis cinerea [62] (52\%). Gar1 has been demonstrated to catalyse the first step of the D-GalUA catabolic pathway, i.e., D-GalUA to L-GalA [48]. The current study demonstrates for the first time that the baker's yeast homologue Gcy1 also shows D-GalUA reducing activity. The fungal D-GalUA reductase Gar1 from $T$. reesei shows a certain flexibility for its substrate and is for example able to convert D,L-glyceraldehyde to glycerol and D-glucuronic acid (D-GlcUA) to L-gulonate [7,48], confirming the broad substrate spectrum of Gcy1 and its homologs. Second, Gcy1 also shows relatively high homology $(41 \%)$ to the last enzyme of the fungal D-GalUA pathway, i.e., from D,L-glyceraldehyde to glycerol (Gld1 from T. reesei or GaaD from A. niger) (Figure 1) [47,63]. This matches the affinity of Gcy1 for D,L-glyceraldehyde (see above). Therefore, it is possible that enzymes of the EC number 1.1.1.365 and 1.1.1.156 share the same evolutionary origin and are all paralogs or orthologs.

Protzko et al. [27] already demonstrated that the presence of a heterologous L-glyceraldehyde reductase is not required for a fully functional D-GalUA pathway since glycerol accumulates in a strain equipped with just the first three enzymes of the fungal D-GalUA pathway. Based on these results, the authors postulated that S. cerevisiae has an endogenous enzyme that can take over the last reaction of the D-GalUA pathway and claimed that Ypr1 is most probably responsible for the conversion of D,L-glyceraldehyde to glycerol in S. cerevisiae. However, since Gcy1 is a paralog of Ypr1 and since it shows a high 
homology to the fungal L-glyceraldehyde reductase, one cannot exclude the possibility that this protein also contributes to this reaction.

Apart from the fact that Gcy1 is responsible for D-GalUA conversion to L-GalA in S. cerevisiae, our data suggest the existence of an additional enzyme in S. cerevisiae that is able to marginally convert D-GalUA to another product. In fact, the HPLC chromatogram of culture supernatants from the gcy 1 deletion strain revealed a peak of increased peak area and so far unknown identity. The appearance of the peak was dependent on the presence of D-GalUA in the culture medium. This peak of unknown identity was almost not detectable in the reference strain (Figure 4). It seems that the absence of Gcy1 facilitates the conversion of D-GalUA to a product different from L-GalA.

The knowledge obtained in the current study will be useful for metabolic engineering endeavours in the yeast $S$. cerevisiae in the context of designing biorefinery concepts for pectin-rich residues resulting from the food industry. In fact, there have been several envisaged applications of the yeast $S$. cerevisiae in this context. As detailed in the introduction, it is obvious to equip baker's yeast with substrate consumption pathways that allow ethanol production from pectin-rich biomass including D-GalUA. However, baker's yeast has also been considered for biotransformations of D-GalUA to meso-galactarate [27] and L-GalA [32,61,64]. Galactarate can be further converted to adipic acid [27,65], and L-GalA is a precursor of L-ascorbic acid (vitamin C) synthesis [66]. A very recent review about the achievements in this field has been provided by Jeong et al. [6]. Depending on the envisaged bioprocess, L-GalA formation from D-GalUA might be either unwanted (if meso-galactarate is the product) or supportive (when baker's yeast is equipped with a pathway in which L-GalA is an intermediate). Respectively, GCY1 deletion or overexpression might be considered.

Supplementary Materials: The following are available online at https:/ /www.mdpi.com/article/ 10.3390/jof7110914/s1, Figure S1: Routes of glycerol catabolism in the glycerol-negative wild-type strain (A) and the two glycerol-positive strains used in this study in order to prove D-galacturonic acid (D-GalUA) conversion to L-galactonate (L-GalA) in synthetic medium and the presence of glycerol (B and C), Figure S2: Comparison of the CEN.PK DHA strain with the reverse engineered CEN.PK strain (CEN.PK RE) in synthetic glycerol medium supplemented with D-GalUA, Table S1: Plasmids used and constructed in this study, Table S2: PCR primers used for constructing and verifying the deletion mutants for gcy $1 \Delta, y p r 1 \Delta$ and gre $3 \Delta$ in the S. cerevisiae strain CEN.PK DHA, Table S3: PCR primers used for the marker replacement in the $2 \mu$-based expression vectors p424GPD and P424GCY1.

Author Contributions: Conceptualization, A.P., D.R., M.K. and E.N.; investigation, D.R., F.L. and A.P.; writing —original draft preparation, D.R.; writing—review and editing, D.R., M.K. and E.N.; supervision, E.N.; project administration, E.N.; funding acquisition, E.N. All authors have read and agreed to the published version of the manuscript.

Funding: This work was funded through the ERA-IB scheme of the 7th EU Framework Program (YEASTPEC; German Federal Ministry of Education and Research, Project No. 031B0267A).

Institutional Review Board Statement: Not applicable.

Informed Consent Statement: Not applicable.

Data Availability Statement: Data are available in this article and as Supplementary Material.

Acknowledgments: We are grateful to Andy Wiranata Wijaya and Atilla Teleki (University of Stuttgart) for kindly conducting the LC-MS analysis for proving the presence or absence of L-GalA in the supernatants of the respective yeast cultivations.

Conflicts of Interest: The authors declare no conflict of interest.

\section{References}

1. Mohnen, D. Pectin structure and biosynthesis. Curr. Opin. Plant Biol. 2008, 11, 266-277. [CrossRef] [PubMed]

2. Edwards, M.C.; Doran-Peterson, J. Pectin-rich biomass as feedstock for fuel ethanol production. Appl. Microbiol. Biotechnol. 2012, 95, 565-575. [CrossRef] 
3. Berlowska, J.; Binczarski, M.; Dziugan, P.; Wilkowska, A.; Kregiel, D.; Witonska, I. Sugar Beet Pulp as a Source of Valuable Biotechnological Products. In Advances in Biotechnology for Food Industry; Elsevier: Amsterdam, The Netherlands, 2018; Volume 14, pp. 359-392, ISBN 9780128114957.

4. John, I.; Muthukumar, K.; Arunagiri, A. A review on the potential of citrus waste for D-Limonene, pectin, and bioethanol production. Int. J. Green Energy 2017, 14, 599-612. [CrossRef]

5. Martins, L.C.; Monteiro, C.C.; Semedo, P.M.; Sá-Correia, I. Valorisation of pectin-rich agro-industrial residues by yeasts: Potential and challenges. Appl. Microbiol. Biotechnol. 2020, 104, 6527-6547. [CrossRef]

6. Jeong, D.; Park, H.; Jang, B.-K.; Ju, Y.; Shin, M.H.; Oh, E.J.; Lee, E.J.; Kim, S.R. Recent advances in the biological valorization of citrus peel waste into fuels and chemicals. Bioresour. Technol. 2021, 323, 124603. [CrossRef]

7. Kuivanen, J.; Biz, A.; Richard, P. Microbial hexuronate catabolism in biotechnology. AMB Express 2019, 9, 16. [CrossRef]

8. Chroumpi, T.; Mäkelä, M.R.; de Vries, R.P. Engineering of primary carbon metabolism in filamentous fungi. Biotechnol. Adv. 2020, 43, 107551. [CrossRef] [PubMed]

9. Hilditch, S.; Bergha, S.; Kalkkinen, N.; Penttila, M.; Richard, P. The Missing Link in the Fungal D-Galacturonate Pathway. J. Biol. Chem. 2007, 282, 26195-26201. [CrossRef] [PubMed]

10. Richard, P.; Hilditch, S. D-Galacturonic acid catabolism in microorganisms and its biotechnological relevance. Appl. Microbiol. Biotechnol. 2009, 82, 597-604. [CrossRef]

11. Biz, A.; Sugai-Guérios, M.H.; Kuivanen, J.; Maaheimo, H.; Krieger, N.; Mitchell, D.A.; Richard, P. The introduction of the fungal D-galacturonate pathway enables the consumption of D-galacturonic acid by Saccharomyces cerevisiae. Microb. Cell Fact. 2016, 15, 144. [CrossRef]

12. Palma, M.; Guerreiro, J.F.; Sá-Correia, I. Adaptive Response and Tolerance to Acetic Acid in Saccharomyces cerevisiae and Zygosaccharomyces bailii: A Physiological Genomics Perspective. Front. Microbiol. 2018, 9, 1-18. [CrossRef]

13. Martins, L.C.; Palma, M.; Angelov, A.; Nevoigt, E.; Liebl, W.; Sá-Correia, I. Complete utilization of the major carbon sources present in sugar beet pulp hydrolysates by the oleaginous red yeasts Rhodotorula toruloides and R. mucilaginosa. J. Fungi 2021, 7, 215. [CrossRef]

14. Huisjes, E.H.; De Hulster, E.; Van Dam, J.C.; Pronk, J.T.; Maris, A.J.A. Van Galacturonic Acid Inhibits the Growth of Saccharomyces cerevisiae on Galactose, Xylose, and Arabinose. Appl. Environ. Microbiol. 2012, 78, 5052-5059. [CrossRef] [PubMed]

15. Van Maris, A.J.A.; Abbott, D.A.; Bellissimi, E.; van den Brink, J.; Kuyper, M.; Luttik, M.A.H.; Wisselink, H.W.; Scheffers, W.A.; van Dijken, J.P.; Pronk, J.T. Alcoholic fermentation of carbon sources in biomass hydrolysates by Saccharomyces cerevisiae: Current status. Antonie Leeuwenhoek 2006, 90, 391-418. [CrossRef] [PubMed]

16. Rezić, T.; Oros, D.; Marković, I.; Kracher, D.; Ludwig, R.; Santek, B. Integrated hydrolyzation and fermentation of sugar beet pulp to bioethanol. J. Microbiol. Biotechnol. 2013, 23, 1244-1252. [CrossRef] [PubMed]

17. Lian, J.; Mishra, S.; Zhao, H. Recent advances in metabolic engineering of Saccharomyces cerevisiae: New tools and their applications. Metab. Eng. 2018, 50, 85-108. [CrossRef] [PubMed]

18. Fraczek, M.G.; Naseeb, S.; Delneri, D. History of genome editing in yeast. Yeast 2018, 35, 361-368. [CrossRef]

19. Moysés, D.; Reis, V.; Almeida, J.; Moraes, L.; Torres, F. Xylose Fermentation by Saccharomyces cerevisiae: Challenges and Prospects. Int. J. Mol. Sci. 2016, 17, 207. [CrossRef]

20. Cunha, J.T.; Romaní, A.; Costa, C.E.; Sá-Correia, I.; Domingues, L. Molecular and physiological basis of Saccharomyces cerevisiae tolerance to adverse lignocellulose-based process conditions. Appl. Microbiol. Biotechnol. 2019, 103, 159-175. [CrossRef]

21. Gonçalves, D.L.; Matsushika, A.; de Sales, B.B.; Goshima, T.; Bon, E.P.S.; Stambuk, B.U. Xylose and xylose/glucose co-fermentation by recombinant Saccharomyces cerevisiae strains expressing individual hexose transporters. Enzyme Microb. Technol. 2014, 63, 13-20. [CrossRef]

22. Krahulec, S.; Petschacher, B.; Wallner, M.; Longus, K.; Klimacek, M.; Nidetzky, B. Fermentation of mixed glucose-xylose substrates by engineered strains of Saccharomyces cerevisiae: Role of the coenzyme specificity of xylose reductase, and effect of glucose on xylose utilization. Microb. Cell Fact. 2010, 9, 16. [CrossRef]

23. Zheng, D.Q.; Wu, X.C.; Tao, X.L.; Wang, P.M.; Li, P.; Chi, X.Q.; Li, Y.D.; Yan, Q.F.; Zhao, Y.H. Screening and construction of Saccharomyces cerevisiae strains with improved multi-tolerance and bioethanol fermentation performance. Bioresour. Technol. 2011, 102, 3020-3027. [CrossRef]

24. Benjaphokee, S.; Hasegawa, D.; Yokota, D.; Asvarak, T.; Auesukaree, C.; Sugiyama, M.; Kaneko, Y.; Boonchird, C.; Harashima, S. Highly efficient bioethanol production by a Saccharomyces cerevisiae strain with multiple stress tolerance to high temperature, acid and ethanol. N. Biotechnol. 2012, 29, 379-386. [CrossRef]

25. Geng, P.; Zhang, L.; Shi, G.Y. Omics analysis of acetic acid tolerance in Saccharomyces cerevisiae. World J. Microbiol. Biotechnol. 2017, 33, 94. [CrossRef] [PubMed]

26. Narendranath, N.V.; Power, R. Relationship between $\mathrm{pH}$ and medium dissolved solids in terms of growth and metabolism of Lactobacilli and Saccharomyces cerevisiae during ethanol production. Appl. Environ. Microbiol. 2005, 71, 2239-2243. [CrossRef] [PubMed]

27. Protzko, R.J.; Latimer, L.N.; Martinho, Z.; de Reus, E.; Seibert, T.; Benz, J.P.; Dueber, J.E. Engineering Saccharomyces cerevisiae for co-utilization of D-galacturonic acid and D-glucose from citrus peel waste. Nat. Commun. 2018, 9, 5059. [CrossRef]

28. Jeong, D.; Ye, S.; Park, H.; Kim, S.R. Simultaneous fermentation of galacturonic acid and five-carbon sugars by engineered Saccharomyces cerevisiae. Bioresour. Technol. 2020, 295, 122259. [CrossRef] [PubMed] 
29. Perpelea, A.; Wijaya, A.W.; Martins, L.C.; Rippert, D.; Klein, M.; Angelov, A.; Peltonen, K.; Teleki, A.; Liebl, W.; Richard, P.; et al. Towards valorization of pectin-rich agro-industrial residues: Engineering of Saccharomyces cerevisiae for co-fermentation of D-galacturonic acid and glycerol. Metab. Eng. 2021, in press. [CrossRef] [PubMed]

30. Souffriau, B.; Den Abt, T.; Thevelein, J.M. Evidence for rapid uptake of D-galacturonic acid in the yeast Saccharomyces cerevisiae by a channel-type transport system. FEBS Lett. 2012, 586, 2494-2499. [CrossRef]

31. Benz, J.P.; Protzko, R.J.; Andrich, J.M.S.; Bauer, S.; Dueber, J.E.; Somerville, C.R. Identification and characterization of a galacturonic acid transporter from Neurospora crassa and its application for Saccharomyces cerevisiae fermentation processes. Biotechnol. Biofuels 2014, 7, 1-13. [CrossRef]

32. Matsubara, T.; Hamada, S.; Wakabayashi, A.; Kishida, M. Fermentative production of L-galactonate by using recombinant Saccharomyces cerevisiae containing the endogenous galacturonate reductase gene from Cryptococcus diffluens. J. Biosci. Bioeng. 2016, 122, 639-644. [CrossRef]

33. Swinnen, S.; Ho, P.W.; Klein, M.; Nevoigt, E. Genetic determinants for enhanced glycerol growth of Saccharomyces cerevisiae. Metab. Eng. 2016, 36, 68-79. [CrossRef]

34. Ho, P.-W.; Klein, M.; Futschik, M.; Nevoigt, E. Glycerol positive promoters for tailored metabolic engineering of the yeast Saccharomyces cerevisiae. FEMS Yeast Res. 2018, 18, foy019. [CrossRef]

35. Ho, P.-W.; Swinnen, S.; Duitama, J.; Nevoigt, E. The sole introduction of two single-point mutations establishes glycerol utilization in Saccharomyces cerevisiae CEN.PK derivatives. Biotechnol. Biofuels 2017, 10, 1-15. [CrossRef]

36. Gietz, R.D.; Schiestl, R.H.; Willems, A.R.; Woods, R.A. Studies on the transformation of intact yeast cells by the LiAc/SSDNA/PEG procedure. Yeast 1995, 11, 355-360. [CrossRef]

37. Gueldener, U.; Heinisch, J.; Koehler, G.J.; Voss, D.; Hegemann, J.H. A second set of loxP marker cassettes for Cre-mediated multiple gene knockouts in budding yeast. Nucleic Acids Res. 2002, 30, e23. [CrossRef]

38. Hoffman, C.S.; Winston, F. A ten-minute DNA preparation from yeast efficiently releases autonomous plasmids for transformaion of Escherichia coli. Gene 1987, 57, 267-272. [CrossRef]

39. Verduyn, C.; Postma, E.; Scheffers, W.A.; Van Dijken, J.P. Effect of benzoic acid on metabolic fluxes in yeasts: A continuous-culture study on the regulation of respiration and alcoholic fermentation. Yeast 1992, 8, 501-517. [CrossRef]

40. Nguyen, H.T.T.; Nevoigt, E. Engineering of Saccharomyces cerevisiae for the production of dihydroxyacetone (DHA) from sugars: A proof of concept. Metab. Eng. 2009, 11, 335-346. [CrossRef] [PubMed]

41. Funk, M.; Niedenthal, R.; Mumberg, D.; Brinkmann, K.; Rönicke, V.; Henkel, T. Vector systems for heterologous expression of proteins in Saccharomyces cerevisiae. Methods Enzymol. 2002, 350, 248-257. [CrossRef] [PubMed]

42. Smith, P.K.; Krohn, R.I.; Hermanson, G.T.; Mallia, A.K.; Gartner, F.H.; Provenzano, M.D.; Fujimoto, E.K.; Goeke, N.M.; Olson, B.J.; Klenk, D.C. Measurement of protein using bicinchoninic acid. Anal. Biochem. 1985, 150, 76-85. [CrossRef]

43. Klein, M.; Swinnen, S.; Thevelein, J.M.; Nevoigt, E. Minireview Glycerol metabolism and transport in yeast and fungi: Established knowledge and ambiguities. Environ. Microbiol. 2016, 19, 878-893. [CrossRef]

44. Klein, M.; Carrillo, M.; Xiberras, J.; Islam, Z.U.; Swinnen, S.; Nevoigt, E. Towards the exploitation of glycerol's high reducing power in Saccharomyces cerevisiae-based bioprocesses. Metab. Eng. 2016, 38, 464-472. [CrossRef]

45. Klein, M.; Islam, Z.u.; Knudsen, P.B.; Carrillo, M.; Swinnen, S.; Workman, M.; Nevoigt, E. The expression of glycerol facilitators from various yeast species improves growth on glycerol of Saccharomyces cerevisiae. Metab. Eng. Commun. 2016, 3, $252-257$. [CrossRef]

46. Swinnen, S.; Klein, M.; Carrillo, M.; McInnes, J.; Nguyen, H.T.; Nevoigt, E. Re-evaluation of glycerol utilization in Saccharomyces cerevisiae: Characterization of an isolate that grows on glycerol without supporting supplements. Biotechnol. Biofuels 2013, 6, 157. [CrossRef]

47. Martens-Uzunova, E.S.; Schaap, P.J. An evolutionary conserved D-galacturonic acid metabolic pathway operates across filamentous fungi capable of pectin degradation. Fungal. Genet. Biol. 2008, 45, 1449-1457. [CrossRef]

48. Kuorelahti, S.; Kalkkinen, N.; Penttilä, M.; Londesborough, J.; Richard, P. Identification in the mold Hypocrea jecorina of the first fungal D-galacturonic acid reductase. Biochemistry 2005, 44, 11234-11240. [CrossRef] [PubMed]

49. Petrash, J.M.; Murthy, B.S.N.; Young, M.; Morris, K.; Rikimaru, L.; Griest, T.A.; Harter, T. Functional genomic studies of aldo-keto reductases. Chem. Biol. Interact. 2001, 130, 673-683. [CrossRef]

50. Chang, Q.; Griest, T.A.; Harter, T.M.; Mark Petrash, J. Functional studies of aldo-keto reductases in Saccharomyces cerevisiae. Biochim. Biophys. Acta Mol. Cell Res. 2007, 1773, 321-329. [CrossRef]

51. Kim, S.T.; Huh, W.K.; Lee, B.H.; Kang, S.O. D-Arabinose dehydrogenase and its gene from Saccharomyces cerevisiae. Biochim. Biophys. Acta 1998, 1429, 29-39. [CrossRef]

52. Hur, E.; Wilson, D.K. Crystallization and aldo-keto reductase activity of Gcy1p from Saccharomyces cerevisiae. Acta Crystallogr. Sect. D Biol. Crystallogr. 2000, 56, 763-765. [CrossRef]

53. Hur, E.; Wilson, D.K. The crystal structure of the GCY1 protein from S. cerevisiae suggests a divergent aldo-keto reductase catalytic mechanism. Chem. Biol. Interact. 2001, 130-132, 527-536. [CrossRef]

54. Liang, Z.; Wang, X.; Bao, X.; Wei, T.; Hou, J.; Liu, W.; Shen, Y. Newly identified genes contribute to vanillin tolerance in Saccharomyces cerevisiae. Microb. Biotechnol. 2021, 14, 503-516. [CrossRef]

55. Blank, L.M.; Lehmbeck, F.; Sauer, U. Metabolic-flux and network analysis in fourteen hemiascomycetous yeasts. FEMS Yeast Res. 2005, 5, 545-558. [CrossRef] [PubMed] 
56. Oechsner, U.; Magdolen, V.; Bandlow, W. A nuclear yeast gene (GCY) encodes a polypeptide with high homology to a vertebrate eye lens protein. FEBS Lett. 1988, 238, 123-128. [CrossRef]

57. Norbeck, J.; Blomberg, A. Metabolic and regulatory changes associated with growth of Saccharomyces cerevisiae in $1.4 \mathrm{M}$ NaCl. J. Biol. Chem. 1997, 272, 5544-5554. [CrossRef] [PubMed]

58. Blomberg, A. Metabolic surprises in Saccharomyces cerevisiae during adaptation to saline conditions: Questions, some answers and a model. FEMS Microbiol. Lett. 2000, 182,1-8. [CrossRef] [PubMed]

59. Roberts, G.G.; Hudson, A.P. Transcriptome profiling of Saccharomyces cerevisiae during a transition from fermentative to glycerolbased respiratory growth reveals extensive metabolic and structural remodeling. Mol. Genet. Genom. 2006, 276, 170-186. [CrossRef]

60. Zhang, L.; Tang, Y.; Guo, Z.; Shi, G. Engineering of the glycerol decomposition pathway and cofactor regulation in an industrial yeast improves ethanol production. J. Ind. Microbiol. Biotechnol. 2013, 40, 1153-1160. [CrossRef]

61. Harth, S.; Wagner, J.; Sens, T.; Choe, J.; Benz, J.P.; Weuster-Botz, D.; Oreb, M. Engineering cofactor supply and NADH-dependent D-galacturonic acid reductases for redox-balanced production of L-galactonate in Saccharomyces cerevisiae. Sci. Rep. 2020, $10,19021$. [CrossRef]

62. Zhang, L.; Thiewes, H.; Kan, J.A.L. Van The D-galacturonic acid catabolic pathway in Botrytis cinerea. Fungal Genet. Biol. 2011, 48, 990-997. [CrossRef] [PubMed]

63. Liepins, J.; Kuorelahti, S.; Penttilä, M.; Richard, P. Enzymes for the NADPH-dependent reduction of dihydroxyacetone and D-glyceraldehyde and L-glyceraldehyde in the mould Hypocrea jecorina. FEBS J. 2006, 273, 4229-4235. [CrossRef] [PubMed]

64. Wagner, J.; Schäfer, D.; von den Eichen, N.; Haimerl, C.; Harth, S.; Oreb, M.; Benz, J.P.; Weuster-Botz, D. D-Galacturonic acid reduction by S. cerevisiae for L-galactonate production from extracted sugar beet press pulp hydrolysate. Appl. Microbiol. Biotechnol. 2021, 105, 5795-5807. [CrossRef] [PubMed]

65. Shiramizu, M.; Toste, F.D. Expanding the scope of biomass-derived chemicals through tandem reactions based on oxorheniumcatalyzed deoxydehydration. Angew. Chemie Int. Ed. 2013, 52, 12905-12909. [CrossRef] [PubMed]

66. Ishikawa, T.; Nishikawa, H.; Gao, Y.; Sawa, Y.; Shibata, H.; Yabuta, Y.; Maruta, T.; Shigeoka, S. The pathway via D-galacturonate/L-galactonate is significant for ascorbate biosynthesis in Euglena gracilis: Identification and functional characterization of aldonolactonase. J. Biol. Chem. 2008, 283, 31133-31141. [CrossRef] [PubMed] 